\title{
Semi-supervised Projection Clustering with Transferred Centroid Regularization
}

\author{
Bin Tong ${ }^{1}, \mathrm{Hao}_{\mathrm{Shao}}{ }^{1}$, Bin-Hui Chou ${ }^{2}$, and Einoshin Suzuki ${ }^{1,2}$ \\ 1 Graduate School of Systems Life Sciences, Kyushu University, Fukuoka, Japan \\ 2 Department of Informatics, ISEE, Kyushu University, Fukuoka, Japan
}

\begin{abstract}
We propose a novel method, called Semi-supervised Projection Clustering in Transfer Learning (SPCTL), where multiple source domains and one target domain are assumed. Traditional semi-supervised projection clustering methods hold the assumption that the data and pairwise constraints are all drawn from the same domain. However, many related data sets with different distributions are available in real applications. The traditional methods thus can not be directly extended to such a scenario. One major challenging issue is how to exploit constraint knowledge from multiple source domains and transfer it to the target domain where all the data are unlabeled. To handle this difficulty, we are motivated to construct a common subspace where the difference in distributions among domains can be reduced. We also invent a transferred centroid regularization, which acts as a bridge to transfer the constraint knowledge to the target domain, to formulate this geometric structure formed by the centroids from different domains. Extensive experiments on both synthetic and practical data sets show the effectiveness of our method.
\end{abstract}

\section{Introduction}

Over the past decades, clustering for high dimensional data, referred as projection clustering, has witnessed an increasing interest in the clustering literature. It is designed to derive a subspace where the clustering performance can be enhanced, and the curse of high dimensionality [18] can be mitigated to some extent as well. The projection clustering can be divided into two categories, which are unsupervised ones 228] and semi-supervised ones [20. An important branch of semi-supervised projection clustering is to exploit additional background information posed on data points, i.e., pairwise constraints, to aid unsupervised projection clustering. Each pairwise constraint indicates whether a pair of data points must reside in the same cluster [21]. However, one limitation of semisupervised projection clustering techniques is that all the data points are drawn from the same distribution. Unfortunately, many scenarios in real applications do not follow this requirement. Typically, many related data sets with different distributions are available, which may be handled by transfer learning [17 13,6].

In this paper, we investigate a new transfer clustering task that would be met in real applications. One example [10] is clustering Web pages from four

J.L. Balcázar et al. (Eds.): ECML PKDD 2010, Part III, LNAI 6323, pp. 306-321, 2010.

(C) Springer-Verlag Berlin Heidelberg 2010 
universities, i.e., Cornell, Texas, Wisconsin and Washington. The Web pages of each university are grouped into 7 categories, i.e., student, faculty, staff, department, course, project and others. The Web pages satisfy the typical setting of transfer learning. The contents from four universities are related to each other. However, their distributions are different, since different universities exhibit different features. In the first three universities, referred as three source domains, we are given pairwise constraints inside each university. We are going to utilize the pairwise constraint knowledge to cluster unlabeled Web pages of Washington university, referred as the target domain. Since the distributions of the source domains are different from that of the target domain, the pairwise constraints from multiple source domains cannot be directly employed in the target domain. Therefore, one challenging issue in this task is how to exploit constraint knowledge from multiple source domains to aid the clustering in the target domain. To the best of our knowledge, this issue has not been addressed in the transfer learning literature.

To handle this challenging issue, we are motivated to invent a novel clustering method, called Semi-supervised Projection Clustering in Transfer Learning (SPCTL), to transfer the constraint knowledge from multiple source domains to the target domain. In the first step, we seek a common subspace where the difference in distributions among the domains can be reduced. We do not expect that the data from different domains have the same cluster structure in the original space, but assume the cluster centroids from all the domains follow a certain manifold structure in the common subspace. That is, similar clusters have similar centroids in the common subspace. For example, as to the faculty clusters from different universities, since the common subspace is spanned by the most relevant features among faculties, the centroids of faculty clusters from different universities are assumed to be located nearby in the common subspace. It has inspired us to invent a transferred centroid regularization to formulate the manifold structure formed by the centroids in the common subspace and to implicitly transfer the constraint knowledge to the target domain.

\section{Problem Setting and Motivation}

The problem setting of our method is described as follows. Suppose that we are given source domains $\mathscr{D}^{s}, s=1,2, \ldots, P(P \geq 2)$, where $P$ is the number of the source domains, and a target domain $\mathscr{D}_{T}$. We define the data set of the source domains as $D_{s}=\left\{\mathbf{x}_{i}^{s}\right\}_{i=1}^{n_{s}}, s=1,2, \ldots, P$, and the data set of the target domain as $D_{T}=\left\{\mathbf{x}_{i}^{\prime}\right\}_{i=1}^{n_{T}}$, where $n_{s}$ denotes the number of data points in the $s$-th source domain, $n_{T}$ represents the number of data points in the target domain, and $\mathbf{x}_{i}^{s}$, $\mathbf{x}_{i}^{\prime} \in \mathbb{R}^{d}$. We assume that there is no irrelevant source domain to the target domain. Let $\mathcal{P}\left(D_{s}\right)$ and $\mathcal{P}\left(D_{T}\right)$ be the marginal distribution of $D_{s}$ and $D_{T}$, respectively. In general, they are somehow related to each other yet different. For the $s$-th source domain $\mathscr{D}_{s}, X_{s}=\left\{D_{s}, D_{T}\right\}$ represents the data points from both the $s$-th source domain and the target domain, and $N_{s}=n_{s}+n_{T}$ represents the number of data points in $X_{s}$. We also assume a set of pairwise constraints for 
each source domain, which are must-link constraints and cannot-link constraints. There is no assumption posed on the pairwise constraints. In general, the pairwise constraints are randomly selected by the users. In the $s$-th source domain, the set of must-link constraints is represented as $\mathbf{M}^{s}=\left\{m_{1}^{s}, m_{2}^{s}, \ldots, m_{r_{s}}^{s}\right\}$, and the set of cannot-link constraints is denoted by $\mathbf{C}^{s}=\left\{c_{1}^{s}, c_{2}^{s}, \ldots, c_{l_{s}}^{s}\right\}$, where $r_{s}$ and $l_{s}$ denote the number of must-link constraints and cannot-link constraints, respectively. More precisely, $m_{i}^{s}$ consists of a pair of points belonging to the same class while $c_{i}^{s}$ consists of a pair of points belonging to different classes. For the target domain, we assume that all the data points are unlabeled. Moreover, we assume that each domain has $K$ clusters, and the $K$ clusters in a domain conceptually correspond to the $K$ clusters in another domain. The output of our method is a cluster indicator matrix $Q_{T}$ for the target domain and a $d \times l$ transformation matrix $W$ with an orthogonal constraint $W^{T} W=I$. $W$ consists of $l$ projective vectors $\left\{\mathbf{w}_{1}, \mathbf{w}_{2}, \ldots, \mathbf{w}_{l}\right\}$, where $l$ is the dimensionality of a common subspace shared by all the domains.

The goal of our method is to obtain a good clustering performance for the target domain in the common subspace. To achieve it, we are going to appropriately transfer the constraint knowledge from multiple source domains $\mathscr{D}_{s}$, $s=1,2, \ldots, P$, to the target domain $\mathscr{D}_{T}$.

\subsection{Motivation for Transferred Centroid Regularization}

In this subsection, we show a motivating example that empirically explains why we build a transferred centroid regularization in a common subspace where the difference in distributions among domains is reduced. We follow the way of [14] to generate five domains, where the first four domains are regarded as the source domains while the last domain is the target domain, as shown from Fig. 1a to Fig. 1e, Each domain consists of examples belonging to one of two classes. The examples of class one (denoted with stars) are drawn from a Gaussian Mixture Model (GMM) and the examples of class two (denoted with circles) are drawn from a single Gaussian. For the domains 1 and 2, the GMM parameters of class one are prescribed by a three-component mixture defined as follows. The mixture weights are $(0.3,0.3,0.4)$; their respective means are $(1,1),(3,3)$ and $(5,5)$; their respective covariance matrices are $\sum_{1}=\left(\begin{array}{ll}0.3 & 0.7 \\ 0.7 & 3.0\end{array}\right), \sum_{2}=\left(\begin{array}{ll}3.0 & 0.0 \\ 0.0 & 0.3\end{array}\right)$ and $\sum_{3}=\left(\begin{array}{cc}3.0 & -0.5 \\ -0.5 & 0.3\end{array}\right)$. The examples of class two are drawn from a single Gaussian with mean $(3.5,0.5)$ and diagonal covariance with symmetric variance 0.5 . For the domains 3, 4 and the target domain, the three component GMM parameters of class one are prescribed as follows. The mixture weights are $(0.3,0.3,0.4)$; their respective means are $(1,1),(3,3)$ and $(5,5)$; their respective covariance matrices are $\sum_{2}, \sum_{3}$ and $\sum_{1}$. The examples of class two are drawn from a single Gaussian with mean $(0.5,3.5)$ and diagonal covariance with symmetric variance 0.5 . Note that each source domain has 50 must-link constraints and 50 cannot-link constraints, which are generated randomly. To keep the 


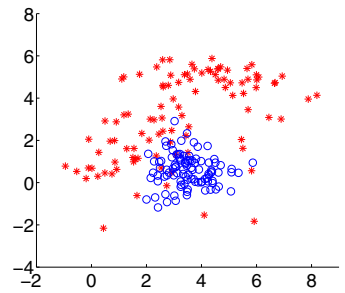

(a) Source Domain 1

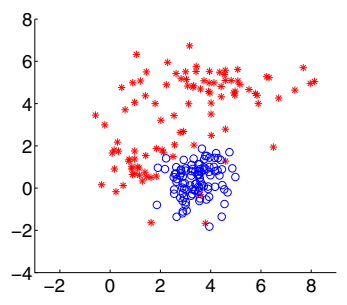

(b) Source Domain 2

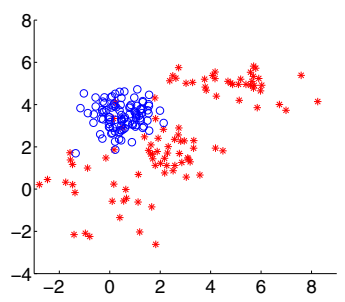

(c) Source Domain 3

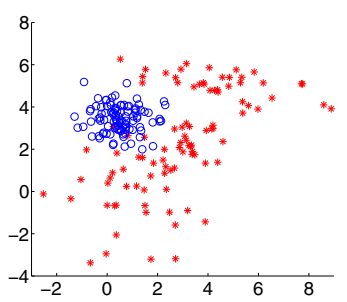

(d) Source Domain 4

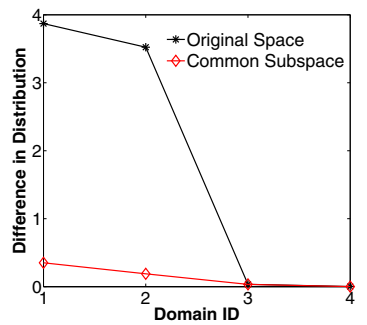

(g) Distribution Difference

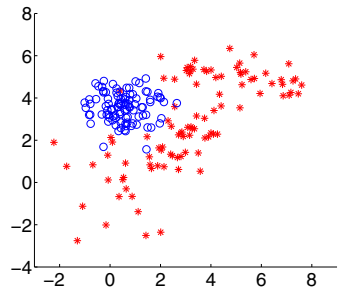

(e) Target Domain

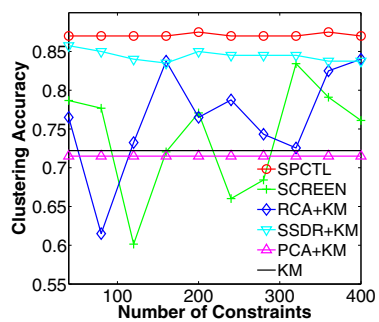

(h) AC

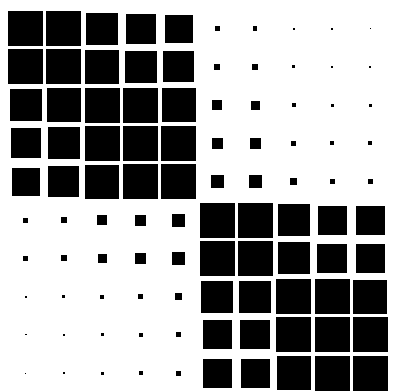

(f) Similarities of Centroids

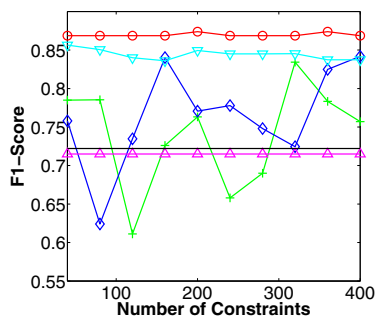

(i) F1-Score

Fig. 1. Motivating example

Figures readable, the pairwise constraints are not shown in the Figures. This motivating example is designed to obtain a one-dimensional common subspace where the clustering for the target domain is performed.

From Fig. 1a to Fig. 1e, we can see that the data between domains 1 and 2 are similar and the data among domains 3, 4 and 5 are similar. However, the distributions between two groups of domains are obviously different. In Fig. 1f, we show that the similarities among ten centroids in the common subspace from all the source domains and the target domain in Hinton diagram [11, in which the square size of the $(i, j)$-th element denotes the degree of similarity between the $i$ th and $j$-th centroids. The similarity is computed by $\exp \left(-\left\|f_{i}-f_{j}\right\|^{2} / 2\right)$, where $f_{i}$ represents the $i$-th centroid. Note that a larger square in the Hinton diagram indicates a larger value of the similarity between two centroids. It is obvious from 
Fig. [1f that the centroids from all the domains form two clusters in the common subspace, each of which is composed of centroids from the corresponding clusters in different domains. This specific geometric distribution of centroids can be considered as a manifold structure. In addition, we employ Maximum Mean Discrepancy (MMD) [16[15] to measure the distribution difference between each source domain and the target domain. As shown in Fig. 1g, we can see that the distribution difference between each source domain and the target domain in the common subspace is much smaller than that in the original space, especially for the domains 1 and 2. In Fig. 1h and Fig. 1il we compare our method, SPCTL, with other methods. Except for $k$-means $(\mathrm{KM})$ and PCA $+k$ means $(\mathrm{PCA}+\mathrm{KM})$, the competitive methods, RCA [1] $+k$ means $(\mathrm{RCA}+\mathrm{KM})$, SSDR $[24]+k$ means (SSDR+KM), and SCREEN [20, simply combine the pairwise constraints from different source domains together. We can see that SPCTL outperforms others in terms of two clustering evaluation measures, clustering accuracy (AC) [23] and F1-Score [6.

From the motivating example, we observe that the centroids from all the domains form a certain manifold structure in the common subspace where the difference in distributions is reduced. This fact has motivated us to embed the constraint knowledge from the source domains into the centroids, and exploit the transferred centroid regularization to transfer the constraint knowledge from multiple source domains to the target domain.

\section{Proposed Framework}

Our framework consists of two steps. In the first step, we invent a new semisupervised projection clustering method which considers the domain adaptation [15]16] and the constraint knowledge. We obtain the centroids, where constraint knowledge is embedded, from all the source domains in the common subspace where the difference in distributions is reduced. In the second step, we propose the transferred centroid regularization to formulate the manifold structure of centroids in the common subspace such that the constraint knowledge can be transferred to the target domain.

\subsection{Projection Clustering}

Before introducing any further, we firstly review a projection clustering method, named AML 22], used in our method. For the $s$-th source domain, each vector $\mathbf{x}_{i}^{s}$ in the $d$-dimensional space is transformed by $W$ to a vector $\mathbf{y}_{i}^{s}$ in the $l$ dimensional space, where $\mathbf{y}_{i}^{s}=W^{T} \mathbf{x}_{i}^{s}$. The distance between two points in the common subspace is measured by the Mahalanobis distance [1] defined as follows:

$$
d_{M}\left(\mathbf{x}_{i}^{s}, \mathbf{x}_{j}^{s}\right)=\sqrt{\left(\mathbf{y}_{i}^{s}-\mathbf{y}_{j}^{s}\right)^{T} \Sigma_{s}^{-1}\left(\mathbf{y}_{i}^{s}-\mathbf{y}_{j}^{s}\right)}
$$

where $\Sigma_{s}$ is the covariance matrix defined as follows:

$$
\Sigma_{s}=\frac{1}{n_{s}} \sum_{i=1}^{n_{s}}\left(\mathbf{y}_{i}^{s}-\mathbf{u}^{s}\right)\left(\mathbf{y}_{i}^{s}-\mathbf{u}^{s}\right)^{T}
$$


where $\mathbf{u}^{s}=\frac{1}{n_{s}} \sum_{i=1}^{n_{s}} \mathbf{y}_{i}^{s}$. In general, maximization of the Sum of Squared Intracluster Error (SSIE) can be equivalently regarded as the cost function of the standard $k$-means algorithm. SSIE is defined as follows:

$$
\mathrm{SSIE}=\sum_{j=1}^{K} n_{s}^{j} d_{M}\left(\mathbf{u}_{j}^{s}, \mathbf{u}^{s}\right)^{2}
$$

where $n_{s}^{j}$ is the sample size of the $j$-th cluster $C_{j}^{s}$ in the $s$-th source domain, and $\mathbf{u}_{j}^{s}$ is the mean of $C_{j}^{s}$. Let $Q_{s}$ be a $n_{s} \times K$ cluster indicator matrix defined as follows. $Q_{s}=\left\{r_{i j}^{s}\right\}_{n_{s} \times K}$, where $r_{i j}^{s}=1$, if $\mathbf{x}_{i}^{s} \in C_{j}^{s}$. We define the weighted cluster indicator matrix as $L_{s}=\left[L_{s}^{1}, L_{s}^{2}, \ldots, L_{s}^{K}\right]$, so that $L_{s}=Q_{s}\left(Q_{s}^{T} Q_{s}\right)^{-\frac{1}{2}}$ [8]. It follows that the $j$-th column of $L_{s}^{j}$ is given by

$$
L_{s}^{j}=(0, \ldots, 0, \overbrace{1, \ldots, 1}^{n_{s}^{j}}, 0, \ldots, 0)^{T} / \sqrt{n_{s}^{j}}
$$

Therefore, SSIE can be succinctly rewritten as the following form [22], which needs to be maximized with respect to $W$ and $L_{s}$ :

$$
\xi_{s}=\frac{1}{n_{s}} \operatorname{tr}\left(L_{s}^{T} D_{s}^{T} W\left(W^{T} \Sigma_{s} W\right)^{-1} W^{T} D_{s} L_{s}\right)
$$

where $t r$ denotes the trace operator.

\subsection{Semi-supervised Projecting Clustering via Domain Adaptation}

The key idea of the new semi-supervised projection clustering is to integrate two factors, which are constraint knowledge and domain adaptation, into projection clustering. Traditional domain adaptation methods only apply to the case of one source domain and one target domain. In our problem setting, we extend it to the case of multiple source domains and one target domain.

As to the $s$-th source domain, following the idea of [12], we change the penalties of violations in the constraints in $\mathbf{M}^{s}$ into the awards. In addition, we place a constraint for all the source domains that the clustering result in the common subspace is the same as that in the original space. The awards in the common subspace can thus be written as follows:

$$
\varphi_{s}=\sum_{\substack{\left\{\mathbf{x}_{i}^{s}, \mathbf{x}_{j}^{s}\right\} \in \mathbf{C}^{s} \\ \text { s.t. } l_{i}^{s}=l_{j}^{s}}} \vartheta_{i j}-\sum_{\substack{\left\{\mathbf{x}_{i}^{s}, \mathbf{x}_{j}^{s}\right\} \in \mathbf{M}^{s} \\ \text { s.t. } l_{i}^{s}=l_{j}^{s}}} \theta_{i j}
$$

where $l_{i}^{s}$ denotes the class label of $\mathbf{x}_{i}^{s}$. Eq. (6), which needs to be minimized with respect to $L_{s}$, can be rewritten as follows:

$$
\varphi_{s}=\operatorname{tr}\left(L_{s}^{T} \Theta^{s} L_{s}\right)
$$

where $\Theta^{s}$ is a $n_{s} \times n_{s}$ matrix with its $(i, j)$-th entry defined as follows:

$$
\Theta_{i j}^{s}=\left\{\begin{array}{cl}
\vartheta_{i j}, & \text { when }\left\{\mathbf{x}_{i}^{s}, \mathbf{x}_{j}^{s}\right\} \in \mathbf{C}^{s} \\
-\theta_{i j}, & \text { when }\left\{\mathbf{x}_{i}^{s}, \mathbf{x}_{j}^{s}\right\} \in \mathbf{M}^{s} \\
0, & \text { otherwise }
\end{array}\right.
$$


where $\vartheta_{i j}$ and $\theta_{i j}$ are both positive reals. To consider the domain adaptation, in the common subspace, we employ Maximum Mean Discrepancy (MMD) [16 15] to measure the mismatch of distributions between the $s$-th source domain and the target domain. The criterion [6], which needs to be minimized with respect to $W$, is defined as follows:

$$
\varpi_{s}=M M D\left[D^{s}, D_{T}\right]=\operatorname{tr}\left(W^{T} X_{s} \mathbf{K}_{s} X_{s}^{T} W\right)
$$

where

$$
\mathbf{K}_{s i j}=\left\{\begin{array}{cl}
\frac{1}{n_{s}^{2}}, & \text { when } \mathbf{x}_{i}^{s}, \mathbf{x}_{j}^{s} \in D_{s} \\
\frac{1}{n_{T}^{2}}, & \text { when } \mathbf{x}_{i}^{\prime}, \mathbf{x}_{j}^{\prime} \in D_{T} \\
\frac{-1}{n_{s} n_{T}}, & \text { otherwise }
\end{array}\right.
$$

So far, we only consider the $s$-th source domain and the target domain. In order to exploit the constraint knowledge from all the source domains and to derive the common subspace shared by all the domains, we then extend the objective function considering all the domain pairs that are composed of each source domain and the target domain. The final objective function, which needs to be minimized with respect to $W$ and $L_{s}$, can be written as follows.

$$
\begin{aligned}
& J=\sum_{s=1}^{P}\left(\varphi_{s}+\alpha \varpi_{s}-\beta \xi_{s}\right) \\
& \text { s.t } W^{T} W=I, L_{s} \in\{0,1\}^{n_{s} \times K}
\end{aligned}
$$

Note that by its definition, the elements of $L_{s}$ can only take binary values, which makes the minimization of Eq. (11) very difficult. Therefore, we relax $L_{s}$ into a nonnegative continuous domain. Then the objective function in Eq. (11) turns out to be

$$
\begin{gathered}
J=\sum_{s=1}^{P}\left(\varphi_{s}+\alpha \varpi_{s}-\beta \xi_{s}\right) \\
\text { s.t } W^{T} W=I, L_{s} \geq 0
\end{gathered}
$$

where both $\alpha$ and $\beta$ are regularization parameters.

\subsection{Target Clustering via Transferred Centroid Regularization}

In the setting of transfer learning, we are more concerned about the performance of the target domain than the source domains. As shown in the previous sections, we assume that the cluster centroids from all the source domains form a certain manifold structure that the centroid of a cluster in a source domain is located nearby with the centroids of the corresponding clusters in other source domains. It is natural assumed that the cluster centroids from the target domain follow the same manifold structure. To exploit the constraint knowledge that is implicitly embedded into the centroids in the common subspace, we thus employ the manifold regularization [2] to formulate this specific structure. 
Since the weighted cluster indicator matrix $L_{s}, s=1,2, \ldots, P$, can be derived from Eq. (12), we can compute the centroid matrix $F_{s}$ by using $L_{s}$. We define $F_{T}$ by the centroid matrix for the target domain. By combining $F_{T}$ with $F_{s}$, $s=1,2, \ldots, P$, we can obtain the centroid matrix for all the domains, denoted by $F=\left[F_{T}, F_{1}, \ldots, F_{P}\right] \in \mathbb{R}^{K \times(P+1)}$. We use $f_{i}$ to denote the $i$-th column of $F$. We then invent the transferred centroid regularization to depict the graph formed by the centroids.

$$
\delta=\frac{1}{2} \sum_{i, j} G_{i j}\left(f_{i}-f_{j}\right)^{2}
$$

where $G$ is a similarity matrix defined as follows:

$$
G_{i j}=\left\{\begin{array}{cl}
\exp \left(\left\|f_{i}-f_{j}\right\|^{2} / \gamma\right), & f_{i} \in N\left(f_{j}\right) \\
0, & \text { otherwise. }
\end{array}\right.
$$

where $N\left(f_{i}\right)$ is defined as the $P$ nearest neighbor set of $f_{i}$. Eq. (13) can be rewritten as follows:

$$
\delta=\operatorname{tr}\left(F^{T} M F\right)
$$

where $M=U-G$ is defined as a graph Laplacian matrix, where $U$ is a diagonal matrix whose entries are column sums of $G, U_{i i}=\sum_{j} G_{i j}$. In the common subspace, we then develop a new cost function for the $k$-means algorithm by integrating the transferred centroid regularization, which is written as the following form:

$$
H=\left\|W^{T} D_{T}-F_{T} Q_{T}^{T}\right\|+\lambda \delta
$$

where $\lambda$ is a regularization parameter. Note that Eq. (16) needs to be minimized. From Eq. (16), our method can been seen as a implicit transfer method, as the constraint knowledge is transferred by formulating the centroid structure.

\subsection{Optimization}

We notice that, for minimizing Eq. (12) with respect to $W$ and $L_{s}, s=1, \ldots, P$, we cannot give a closed-form solution. However, Eq. (12) can be iteratively optimized with respect to $L_{s}$ by fixing $W$, and vice versa.

Specially, when $W$ is fixed, optimizing Eq. (12) with respect to $L_{s}$ is equivalent to optimizing

$$
\begin{aligned}
& J_{L_{s}}=\operatorname{tr}\left(L_{s}^{T} \Theta^{s} L_{s}\right)-\frac{\beta}{n_{s}} \operatorname{tr}\left(L_{s}^{T}\left(D_{s}^{T} W\left(W^{T} \Sigma_{s} W\right)^{-1} W^{T} D_{s}\right) L_{s}\right) \\
& \text { s.t. } L_{s} \geq 0
\end{aligned}
$$

We introduce the Lagrangian multiplier $\psi \in \mathbb{R}^{n_{s} \times K}$, and the Lagrangian function is

$$
\begin{aligned}
J_{L_{s}}^{*} & =\operatorname{tr}\left(L_{s}^{T} \Theta^{s} L_{s}\right)-\frac{\beta}{n_{s}} \operatorname{tr}\left(L_{s}^{T}\left(D_{s}^{T} W\left(W^{T} \Sigma_{s} W\right)^{-1} W^{T} D_{s}\right) L_{s}\right) \\
& -\operatorname{tr}\left(\psi L_{s}^{T}\right)
\end{aligned}
$$


By setting $\frac{\partial J_{L_{s}}^{*}}{\partial L_{s}}=0$, we obtain

$$
\psi=2 \mathbf{A} L_{s}
$$

where $\mathbf{A}=\Theta^{s}-\frac{\beta}{n_{s}}\left[D_{s}^{T} W\left(W^{T} \Sigma_{s} W\right)^{-1} W^{T} D_{s}\right]$. Using the Karush-Kuhn-Tucker condition [5] $\psi_{i j} L_{s_{i j}}=0$, we get

$$
\left[\mathbf{A} L_{s}\right]_{i j} L_{s_{i j}}=0
$$

By introducing $\mathbf{A}=\mathbf{A}^{+}-\mathbf{A}^{-}$where $\mathbf{A}^{+}=\left(\left|\mathbf{A}_{i j}\right|+\mathbf{A}_{i j}\right) / 2$ and $\mathbf{A}^{-}=\left(\left|\mathbf{A}_{i j}\right|-\right.$ $\left.\mathbf{A}_{i j}\right) / 2[9$, we obtain

$$
\left[\mathbf{A}^{+} L_{s}-\mathbf{A}^{-} L_{s}\right]_{i j} L_{s_{i j}}=0
$$

Eq. (21) leads to the following updating formula.

$$
L_{s_{i j}} \leftarrow L_{s_{i j}} \sqrt{\frac{\left[\mathbf{A}^{-} L_{s}\right]_{i j}}{\left[\mathbf{A}^{+} L_{s}\right]_{i j}}}
$$

Eq. (17) can be proven to be non-increasing by using the auxiliary function approach [9]. Due to space limitation, we leave it to the later version of our paper.

When $L_{s}$ is fixed, optimizing Eq. (12) with respect to $W$ is equivalent to optimizing

$$
\begin{aligned}
& J_{W}=\sum_{s=1}^{P}\left[\alpha \operatorname{tr}\left(W^{T} \mathbf{G}_{s} W\right)-\frac{\beta}{n_{s}} \operatorname{tr}\left(\left(W^{T} \mathbf{C}_{s} W\right)^{-1} W^{T} \mathbf{E}_{s} W\right)\right] \\
& \text { s.t. } W^{T} W=I
\end{aligned}
$$

where $\mathbf{G}_{s}=X_{s} \mathbf{K}_{s} X_{s}^{T}, \mathbf{C}_{s}=\Sigma_{s}$, and $\mathbf{E}_{s}=D_{s} L_{s} L_{s}^{T} D_{s}^{T}$. We use the gradient descent to obtain the optimal $W$ for Eq.(23). Starting with an initial $W^{0}$, the gradient descent method successively updates $W$ by

$$
W^{t}=W^{t-1}-\left.v \frac{\partial J_{W}}{\partial W}\right|_{W=W^{t-1}}
$$

where

$$
\begin{aligned}
\frac{\partial J_{W}}{\partial W} & =2 \alpha \sum_{s=1}^{P}\left(\mathbf{G}_{s} W\right)+2 \sum_{s=1}^{P}\left[\frac{\beta}{n_{s}} \mathbf{C}_{s} W\left(W^{T} \mathbf{C}_{s} W\right)^{-1} W^{T} \mathbf{E}_{s} W\left(W^{T} \mathbf{C}_{s} W\right)^{-1}\right] \\
& +2 \sum_{s=1}^{P}\left[\frac{\beta}{n_{s}} \mathbf{E}_{s} W\left(W^{T} \mathbf{C}_{s} W\right)^{-1}\right]
\end{aligned}
$$

where $v$ is the step size and $t$ is the iteration number. We also notice that the updating rule of Eq. (24) is given without the orthogonality constraint $W^{T} W=$ $I$. After each updating step of $W^{t}$, let $W^{t}=W^{t^{\prime}} R$ be the $Q R$ decomposition [5] of $W^{t}$, where $W^{t^{\prime}}$ has orthogonal columns and $R$ is an upper triangle. $W^{t}$ is then replaced by $W^{t^{\prime}}$ for the next iteration. Eq. (23) is iteratively solved until a convergence condition, which is explained in detail in the next subsection, is satisfied. 


\subsection{Clustering Framework}

This clustering framework mainly includes two objective functions, which are Eq.(12) and Eq.(16). Eq.(12) is designed to obtain the common subspace and the weighted cluster indicator matrix $L_{s}, s=1,2, \ldots, P$. The optimization of Eq. (12) is achieved by both Eq. (17) and Eq. (23). In Eq.(16), besides the cost function of the standard $k$-means algorithm, the transferred centroid regularization is taken into consideration. The cluster indicator matrix $Q_{T}$ of the target domain can be derived through the standard $k$-means optimization by using Eq.(16). After obtaining $Q_{T}$, the LDA criterion is employed to select the most discriminative subspace. The optimizations for Eq.(12), Eq. (23) and Eq.(16) are adaptively performed until the convergence is reached. Here, we define a function, named IsConvergent $(\rho, l)$, to determine whether the convergence condition $\left|\rho_{l}-\rho_{l-1}\right| /\left|\rho_{l-1}\right|<\epsilon$ holds, where $l$ is the iteration number, $\rho_{l}$ is the value of the objective function in the $l$-th iteration, and $\epsilon$ is set to be 0.05 . The main steps of clustering framework are presented in Algorithm 1. Although the whole framework is optimized by iterative steps, the empirical result shows it converges fast and iteration times for $t_{1}, t_{2}$ and $t$ are less than five in most cases.

\section{Evaluation by Experiments}

\subsection{Experiments Setup}

We performed experiments on the 20 Newsgroups corpus ${ }^{1}$, which consists of approximately 20000 newsgroup documents collected evenly from 20 different newsgroups. The documents from some different newsgroups are related. For example, the newsgroups rec.sport.baseball and rec.sport.hockey are relevant to recreation. According to the typical setting of transfer learning, we employed the strategy from [6] to construct the data sets in the following way. First, we applied the typical pre-processing steps [19: (1) removed stop words; (2) ignored file headers; (3) selected the top words by mutual information. In our experiments, we selected the top 100 words by ranking the mutual information value of each word. For each domain, we then randomly selected 200 documents for a given upper level category, i.e., comp, rec, sci, and talk. Specific details of the data sets are described in Table 1 . We denote the data set NG1- $i(i=1,2,3)$ by the data set where the $i$-th domain from NG1 is regarded as the target domain and the remaining domains are the source domains. This specification also applies to the data set NG2- $i(i=1,2,3)$.

In our experiments, we compare SPCTL with the following typical methods: $k$-means (KM), PCA+KM, SSDR 24]+KM, RCA [1]+KM, SCREEN [20]. We also use two metrics, the clustering accuracy (AC) 23] and F1-Score [6], to measure the clustering performance. Since SSDR+KM, RCA+KM and SCREEN belong to the semi-supervised methods exploiting the pairwise constraints, for these three methods, we simply combine the data from all the domains together

\footnotetext{
${ }^{1}$ http://people.csail.mit.edu/jrennie/20Newsgroups/
} 


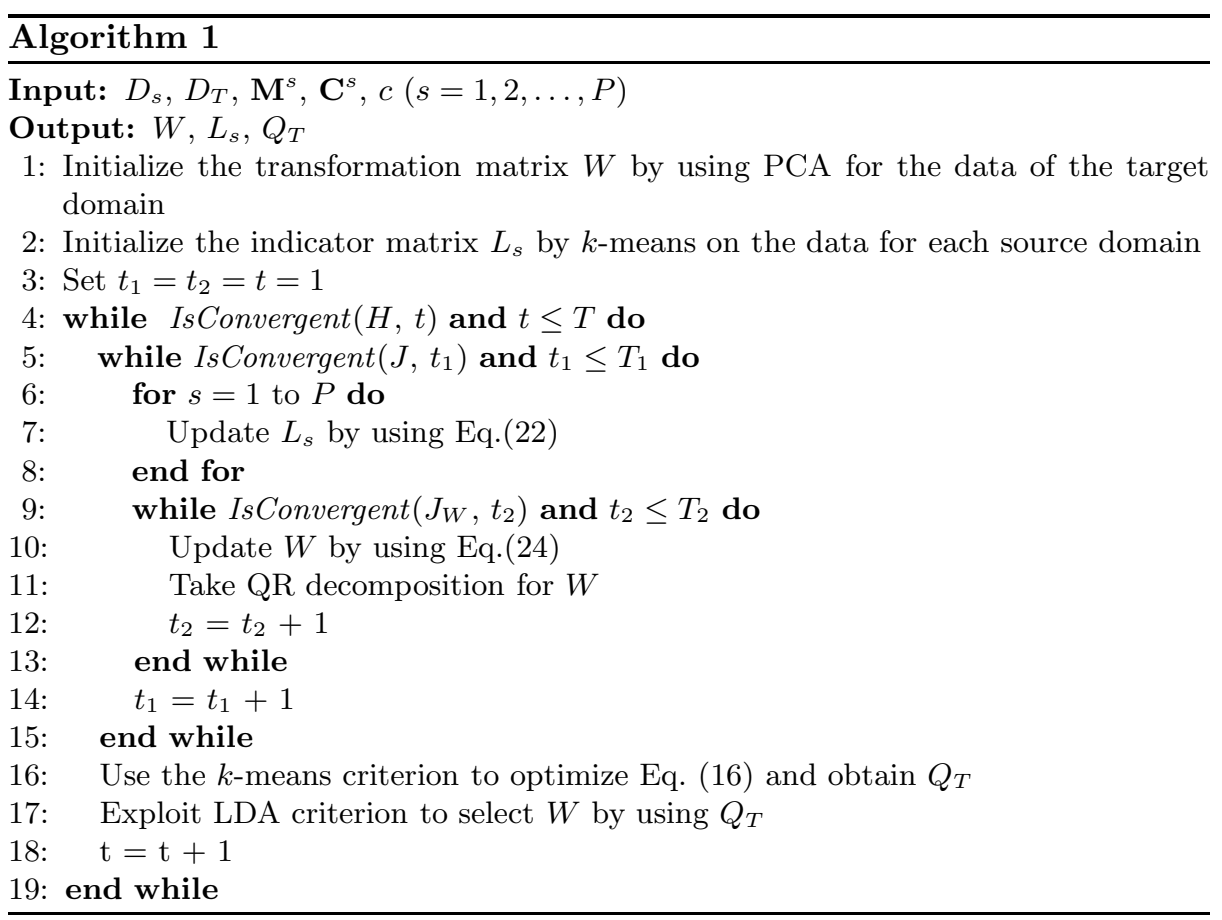

and the pairwise constraints from all the source domains. However, we only consider the data from target domain when computing ACC and F1. For KM and $\mathrm{PCA}+\mathrm{KM}$, we directly applied them to the target domain. For each data set, we repeated the experiments for 10 trails, and report the averages.

The parameter setting of SPCTL is listed as follows. The Gaussian kernel parameter $\gamma$, which is used to construct the transferred centroid regularization, is set to be 10. Since our method is iterative, we prescribe the maximum number of iterations as $T=T_{1}=T_{2}=10$. The parameters $\vartheta$ and $\theta$, which are used in Eq. (8) to code the information of pairwise constraints, are both set to be 0.5. The step size $v$ for updating $W$ is set to be 0.01 . The balancing parameters $\alpha, \beta$, and $\lambda$ are set by searching the grid $\left\{0,10^{0}, 10^{1}, 10^{2}, 10^{3}\right\}$. In order to compare methods fairly, for the parameter settings of other competitive methods, we follow the parameters recommended by them, which are considered to be optimal. Without specific explanation, each source domain has the same number of pairwise constraints. The number of must-link constraints is always set to be equal to that of cannot-link constraints. Must-link constraints and cannot-link constraints are randomly selected according to the ground-truth of class labels.

\subsection{Analysis of Experiments}

In this subsection, we show the effectiveness of SPCTL. First, our performance is exhibited by varying the number of constraints when the reduced dimension is fixed. The reduced dimension for all the data sets is set to be 50. As illustrated 
Table 1. Statistics for our data sets from Newsgroup corpus

\begin{tabular}{|c|c|c|c|c|c|}
\hline \multirow[b]{2}{*}{ Dataset } & \multirow[b]{2}{*}{ Domain } & \multicolumn{3}{|c|}{ Class label } & \multirow[b]{2}{*}{ \#doc } \\
\hline & & comp & sci & talk & \\
\hline \multirow{4}{*}{ NG1 } & 1 & graphics & crypt & $\mathrm{N} / \mathrm{A}$ & 600 \\
\hline & 2 & os.ms-windows.misc motorcycles & electronics & $\mathrm{N} / \mathrm{A}$ & 600 \\
\hline & 3 & sys.ibm.pc.hardware sport.basebal & med & $\mathrm{N} / \mathrm{A}$ & 600 \\
\hline & 4 & sys.mac.hardware & space & $\mathrm{N} / \mathrm{A}$ & 600 \\
\hline \multirow{3}{*}{ NG2 } & 1 & ibm.pc.hardware & electronics & politics.guns & 800 \\
\hline & 2 & sys.mac.hardware sport.basebal & med & olitics.mideast & 800 \\
\hline & 3 & os.ms-windows.misc sport.hockey & crypt & politics.misc & 800 \\
\hline
\end{tabular}

in Fig. 2, in most cases, SPCTL keeps the best performance when the number of available constraints from each source domain increases from 10 to 100. The performances of the three semi-supervised methods are inferior to that of SPCTL. A possible reason is the direct use of data and pairwise constraints from source domains without considering the distribution difference would not guarantee a good performance. As shown in Fig. 2c and Fig. 2d, the performances of SCREEN are fluctuated. The reason is probably that the dimension reduction step of SCREEN depends only on the new generated cannot-link constraints. Because the pairwise constraints are randomly selected, the new generated cannot-link constraints are more likely to be affected by the random selection, so that the subspace is largely influenced by the randomness of the new generated cannotlink constraints. It can be seen from Fig. $2 \mathrm{~g}$ and Fig. $2 \mathrm{~h}$ that SPCTL is unable to outperform others. The most probable reason is that SPCTL fails to obtain the optimal common subspace, so that the transferred centroid regularization behaves inappropriately.

Second, we show the performance of SPCTL when the reduced dimension varies, i.e., 80, 40, 20, and 10, and the average performances over the four dimensions are also presented. In this experiment, we set the number of pairwise constraints to be 100 for each source domain. As shown in Fig. 3, it can be seen that, in most cases, the average performances of SPCTL over different reduced dimensions are kept the highest, indicating that SPCTL is robust against the change of the reduced dimensions. In addition, although $\mathrm{PCA}+\mathrm{KM}$ is slightly better than the standard $k$-means, $\mathrm{PCA}+\mathrm{KM}$ is in general not competitive, probably because the constraint knowledge is not used. We also observe that, in most cases, the average performances of $\mathrm{RCA}+\mathrm{KM}$ and $\mathrm{SSDR}+\mathrm{KM}$ are better than those of $\mathrm{PCA}+\mathrm{KM}$. The possible reason is, although the pairwise constraints come from different domains, they are still informative in the subspace selection. The reason that $\mathrm{RCA}+\mathrm{KM}$ and $\mathrm{SSDR}+\mathrm{KM}$ are inferior to SCREEN is probably that they were not proposed for a clustering task. It is not surprising that SPCTL outperforms SCREEN in most cases. It consists in the fact that they behave in a different way of exploiting constraint knowledge from multiple source domains. This validates our conjecture that transferring constraint knowledge by using the transferred centroid regularization is more effective than the way of simply combining the pairwise constraints. 


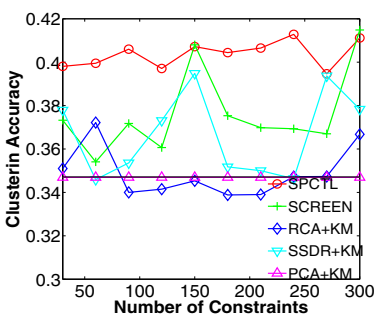

(a) NG1-1 AC

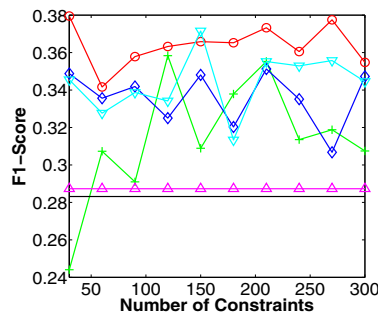

(d) NG1-2 F1-Score

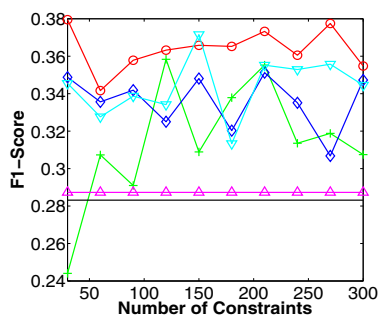

(g) NG2-1 AC

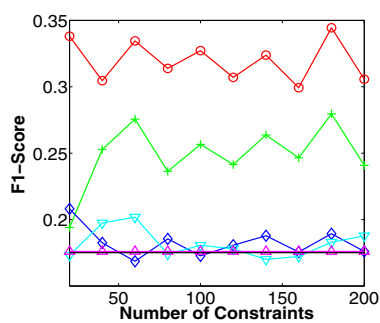

(j) NG2-2 F1-Score

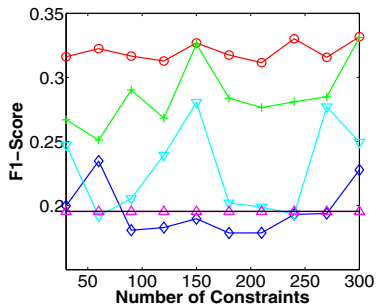

(b) NG1-1 F1-Score

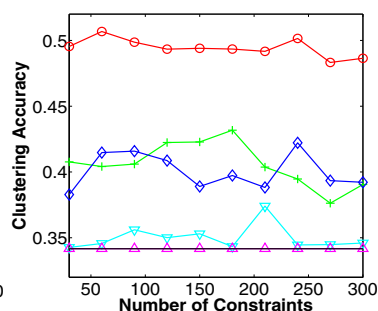

(e) NG1-3 AC

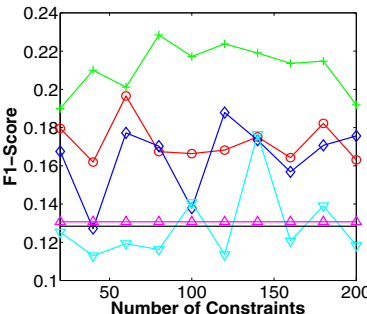

(h) NG2-1 F1-Score

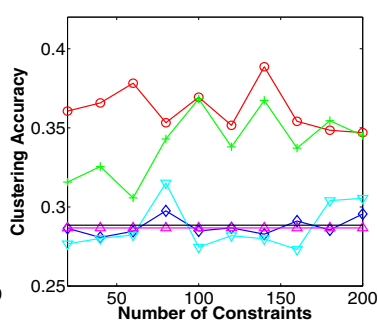

(k) NG2-3 AC

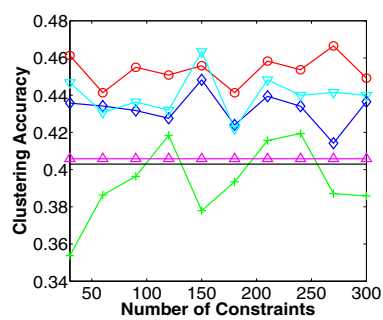

(c) NG1-2 AC

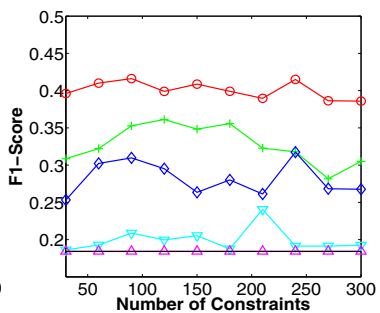

(f) NG1-3 F1-Score

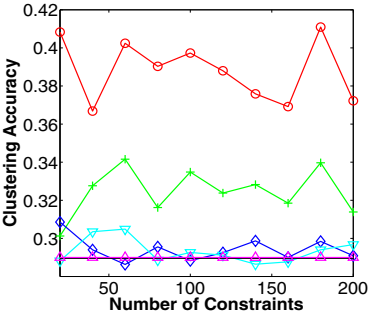

(i) NG2-2 AC

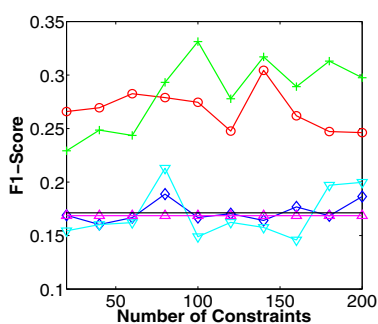

(l) NG2-3 F1-Score

Fig. 2. The performance with different numbers of constraints 


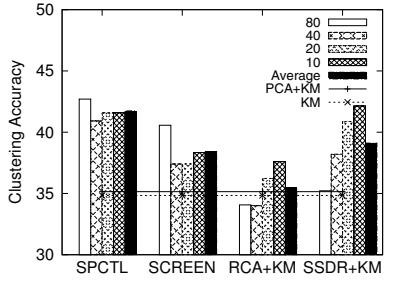

(a) NG1-1 AC

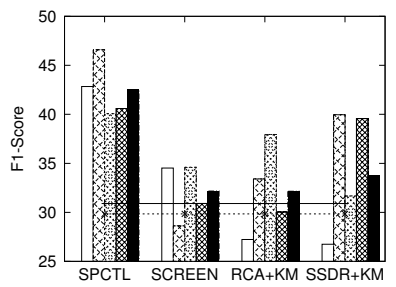

(d) NG1-2 F1-Score

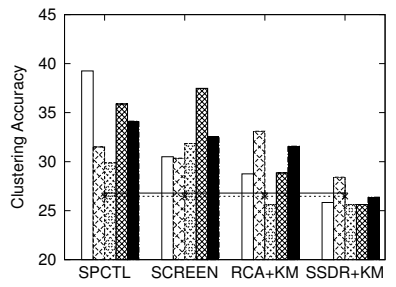

(g) NG2-1 AC

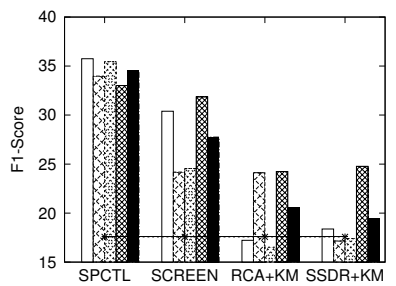

(j) NG2-2 F1-Score

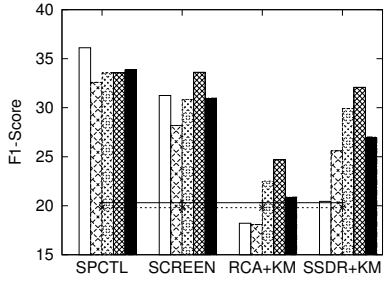

(b) NG1-1 F1-Score

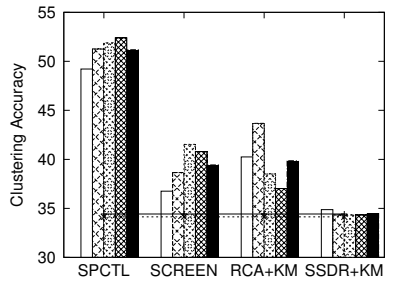

(e) NG1-3 AC

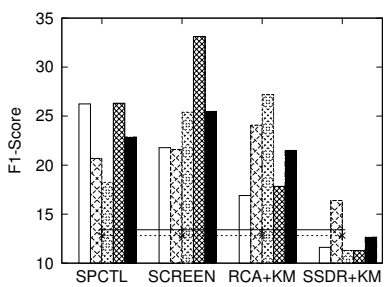

(h) NG2-1 F1-Score

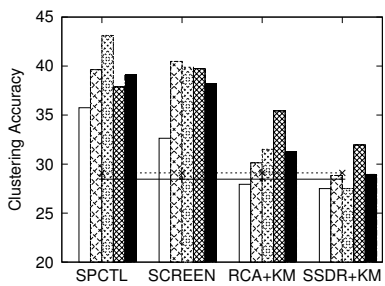

(k) NG2-3 AC

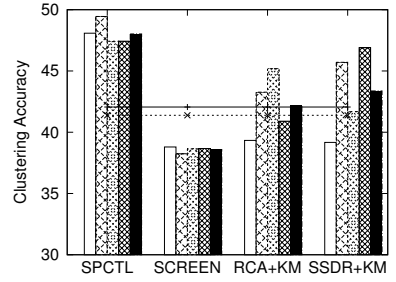

(c) NG1-2 AC

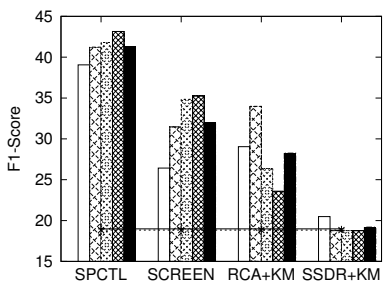

(f) NG1-3 F1-Score

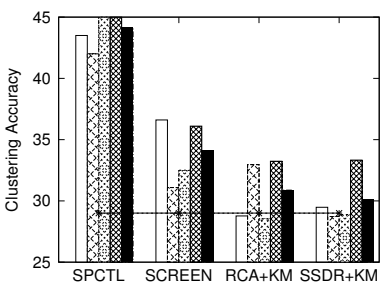

(i) NG2-2 AC

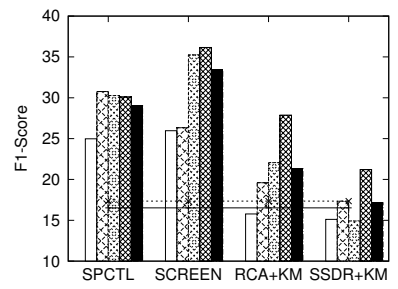

(1) NG2-3 F1-Score

Fig. 3. The performance with different dimensions

\section{Related Works}

In this section, we review past research works related to our work, including semi-supervised clustering, domain adaptation and transfer clustering.

Semi-supervised clustering can be traced back to [21], where pairwise constraints were employed to aid the unsupervised clustering. Semi-supervised clustering for high dimensional data can be implemented by metric-based methods 
201124. However, semi-supervised clustering assumes the data are drawn from the same domain.

Domain adaptation is aimed to obtain a good feature representation that is able to reduce the difference in distributions between domains. Domain adaptation is one of the most significant issues in transfer learning, because different domains would probably cause the negative transfer [17. Blitzer et al. 4] proposed a Structural Correspondence Learning (SCL) method to select domain independent pivot features that appear frequently in different domains, so that the same feature structure can be shared. Recently, Pan et al. 15 invented a new dimension reduction method, named MMDE, for domain adaptation by exploiting the Maximum Mean Discrepancy (MMD) criterion. MMDE is extended in 16 to the out-of-sample case, and reduced the computational time. However, in these two methods, the final classification tasks were independent from the domain adaptation. Chen et al. [6] employed the domain adaptation method to extend a classification framework for transfer learning. It is different from our task setting as our task is for clustering.

Our work is closely related to the transfer clustering of which goal is to improve the clustering performance of the target domain. Dai et al. [7] proposed a Self-taught Clustering (STC) method that aims at clustering a small collection of target unlabeled data with the help of a large amount of auxiliary unlabeled data from source domains. Bhattacharya et al. 3 invented a cross-guided clustering method, in which the clustering partitions of the source domains are manually prescribed. The cross-domain distance measure is then designed to aid the alignment for centroids across domains. Unlike these methods, the input of our transfer clustering task includes pairwise constraints.

\section{Conclusions and Future Works}

In this paper, we investigated a new task of handling the semi-supervised projection clustering in the transfer learning setting. In this task, we carefully designed an iterative framework to obtain a common subspace where the difference in distributions among domains is reduced. Inspired by the specific structure formed by the centroids in the common subspace, we transfer the constraint knowledge to the target domain by inventing the transferred centroid regularization, such that the clustering performance of the target domain in the common subspace can be enhanced. The experimental results show that our method is effective.

Although we have obtained the common subspace where the distribution differences between each source domain and the target domain are reduced, the analysis to the similarities between source domains in the common subspace is not sufficiently discussed. Therefore, in the future work, we are interested in incorporating the similarities between source domains into our framework.

Acknowledgments. This work is partially supported by the grant-in-aid for scientific research on fundamental research (B) 21300053 from the Japanese Ministry of Education, Culture, Sports, Science and Technology. Bin Tong and Hao Shao are sponsored by the China Scholarship Council (CSC). 


\section{References}

1. Bar-Hillel, A., Hertz, T., Shental, N., Weinshall, D.: Learning a Mahalanobis Metric from Equivalence Constraints. J. Mach. Learn. Res. 6, 937-965 (2005)

2. Belkin, M., Niyogi, P., Sindhwani, V.: Manifold Regularization: A Geometric Framework for Learning from Labeled and Unlabeled Examples. J. Mach. Learn. Res. 7, 2399-2434 (2006)

3. Bhattacharya, I., Godbole, S., Joshi, S., Verma, A.: Cross-Guided Clustering: Transfer of Relevant Supervision across Domains for Improved Clustering. In: ICDM, pp. 41-50 (2009)

4. Blitzer, J., McDonald, R., Pereira, F.: Domain Adaptation with Structural Correspondence Learning. In: EMNLP, pp. 120-128 (2006)

5. Boyd, S., Vandenberghe, L.: Convex Optimization. Cambridge University Press, Cambridge (March 2004)

6. Chen, B., Lam, W., Tsang, I., Wong, T.L.: Extracting Discriminative Concepts for Domain Adaptation in Text Mining. In: KDD, pp. 179-188 (2009)

7. Dai, W., Yang, Q., Xue, G.R., Yu, Y.: Self-taught Clustering. In: ICML, pp. 200-207 (2008)

8. Ding, C., He, X., Simon, H.D.: On the Equivalence of Nonnegative Matrix Factorization and Spectral Clustering. In: SDM (2005)

9. Ding, C., Li, T., Jordan, M.I.: Convex and Semi-Nonnegative Matrix Factorizations. IEEE Trans. PAMI 32, 45-55 (2010)

10. Gu, Q., Zhou, J.: Learning the Shared Subspace for Multi-task Clustering and Transductive Transfer Classification. In: ICDM, pp. 159-168 (2009)

11. Hinton, G.E., Sejnowski, T.J.: Learning and Relearning in Boltzmann Machines, pp. 282-317 (1986)

12. Kulis, B., Basu, S., Dhillon, I., Mooney, R.: Semi-supervised Graph Clustering: A Kernel Approach. In: ICML, pp. 457-464 (2005)

13. Ling, X., Dai, W., Xue, G.R., Yang, Q., Yu, Y.: Spectral Domain-transfer Learning. In: KDD, pp. 488-496 (2008)

14. Liu, Q., Liao, X., Carin, H.L., Stack, J.R., Carin, L.: Semisupervised Multitask Learning. IEEE Trans. PAMI 31(6), 1074-1086 (2009)

15. Pan, S.J., Kwok, J.T., Yang, Q.: Transfer Learning via Dimensionality Reduction. In: AAAI, pp. 677-682 (2008)

16. Pan, S.J., Tsang, I.W., Kwok, J.T., Yang, Q.: Domain Adaptation via Transfer Component Analysis. In: IJCAI, pp. 1187-1192 (2009)

17. Pan, S.J., Yang, Q.: A Survey on Transfer Learning. IEEE Trans. TKDE 99 (2009)

18. Parsons, L., Haque, E., Liu, H.: Subspace Clustering for High Dimensional Data: A Review. SIGKDD Explor. Newsl. 6(1), 90-105 (2004)

19. Slonim, N., Tishby, N.: Document Clustering Using Word Clusters via the Information Bottleneck Method. In: SIGIR, pp. 208-215 (2000)

20. Tang, W., Xiong, H., Zhong, S., Wu, J.: Enhancing Semi-supervised Clustering: A Feature Projection Perspective. In: KDD, pp. 707-716 (2007)

21. Wagstaff, K., Cardie, C.: Clustering with Instance-level Constraints. In: ICML, pp. 1103-1110 (2000)

22. Ye, J., Zhao, Z., Liu, H.: Adaptive Distance Metric Learning for Clustering. In: CVPR (2007)

23. Ye, J., Zhao, Z., Wu, M.: Discriminative K-means for Clustering. In: NIPS (2007)

24. Zhang, D., Zhou, Z., Chen, S.: Semi-supervised Dimensionality Reduction. In: SDM (2007) 